EVALUATING THE PROBABILITY OF VIOLATING DISSOLVED OXYGEN STANDARD

Y.K. Tung

1990

Journal Article

WWRC $-90-18$

\author{
In \\ Ecological Modelling \\ Volume 51 \\ Y.K. Tung \\ Wyoming Water Research Center \\ and \\ Statistics Department \\ University of Wyoming \\ Laramie, Wyoming
}




\title{
EVALUATING THE PROBABILITY OF VIOLATING DISSOLVED OXYGEN STANDARD
}

\author{
YEOU-KOUNG TUNG \\ Wyoming Water Research Center and Statistics Department, University of Wyoming, Laramie, \\ WY 82071 (U.S.A.)
}

(Accepted 15 November 1989)

\begin{abstract}
Tung, Y.K., 1990. Evaluating the probability of violating dissolved oxygen standard. Ecol. Modelling, 51: 193-204.

One of the main concerns in water quality control and regulation is the assurance of compliance of the specified water quality standard. Due to the existence of various uncertainties in a stochastic stream environment, such compliance cannot be guaranteed at all times. This paper discussed and applies methods for evaluating the risk of violating the water quality standard. The methodologies are applied to evaluate the risk of violating dissolved oxygen standard using the Streeter-Phelps dissolved oxygen model.
\end{abstract}

\section{INTRODUCTION}

In water quality control and regulation, one of the main concerns is the assurance of water quality compliance to the specified standards. To predict and evaluate the impacts of the various decisions on water quality, models are frequently used. Due to the existence of various uncertainties in stream environments and modeling processes the true impact cannot be assessed with absolute certainty. Therefore, the compliance of a specified water quality standard cannot be guaranteed. As part of the evaluation criteria, the merit of a water quality decision should be assessed by the likelihood of occurrence of adverse water quality conditions caused by violating the standard.

This paper discusses several methodologies to evaluate the risk of violating the specified water quality standard using a water quality model. Emphasis is placed on the comparison of two first-order second moment methods. For demonstration, the the instream dissolved oxygen (DO) concentration is adopted as the indicator of water quality condition. The Streeter-Phelps (1925) equation will be utilized throughout the paper to 
discuss the methods. The risk evaluation techniques are applicable to any water quality models.

\section{USE OF DISSOLVED OXYGEN MODELS}

To evaluate the impacts of water quality management decisions, models that describe the relationships between water quality condition and decision are frequently used. Dissolved oxygen models describe the impact of waste discharge on the instream DO concentration. Since the first DO model introduced by Streeter and Phelps (1925) many modifications and extensions were made to include various oxygen sources and sinks (Dobbins, 1964; Krenkel and Novotny, 1980).

Using the Streeter-Phelps model, the Do deficit concentration can be estimated as:

$D_{x}=\frac{K_{\mathrm{a}} L_{0}}{K_{\mathrm{a}}-K_{\mathrm{d}}}\left[\exp \left(-K_{\mathrm{d}} x / U\right)-\exp \left(-K_{\mathrm{a}} x / U\right)\right]+D_{0} \exp \left(-K_{\mathrm{a}} x / U\right)$

where $D_{x}$ is the Do deficit concentration $(\mathrm{mg} / \mathrm{L})$ at downstream distance $x$ miles from the initial point $(x=0), K_{\mathrm{d}}$ and $K_{\mathrm{a}}$ are deoxygenation and reaeration coefficients (both in day ${ }^{-1}$ ), respectively, $U$ is the average flow velocity (miles/day), and $L_{0}$ and $D_{0}$ are, respectively, the initial BOD and DO deficit concentrations at $x=0$ (both in $\mathrm{mg} / \mathrm{L}$ ). The DO concentrations $C_{x}(\mathrm{mg} / \mathrm{L})$ at any downstream location can be obtained as:

$C_{x}=C_{\text {sat }}-D_{x}$

in which $C_{\text {sat }}$ is the saturated Do concentration $(\mathrm{mg} / \mathrm{L})$.

\section{UNCERTAINTIES IN WATER QUALITY MODELS}

Using a model deterministically in water quality decision-making implies that potential impacts on stream water quality condition by a given management scenario can be assessed definitely by the model. In fact, there exist several uncertainties in water quality modeling (Hathhorn and Tung, 1988) including inherent, model and parameter uncertainties.

Inherent uncertainty is referred to as the random characteristics of pollutant transport process in natural streams. Physical, biological and chemical properties of dominant factors affecting water quality in the streams are constantly changing with respect to time and space.

Model uncertainty has resulted from the use of an idealized model to describe the complex phenomena involved in pollutant transport processes. For example, the Streeter-Phelps equation is a simplified water quality model which does not consider other potential oxygen sources and sinks. 


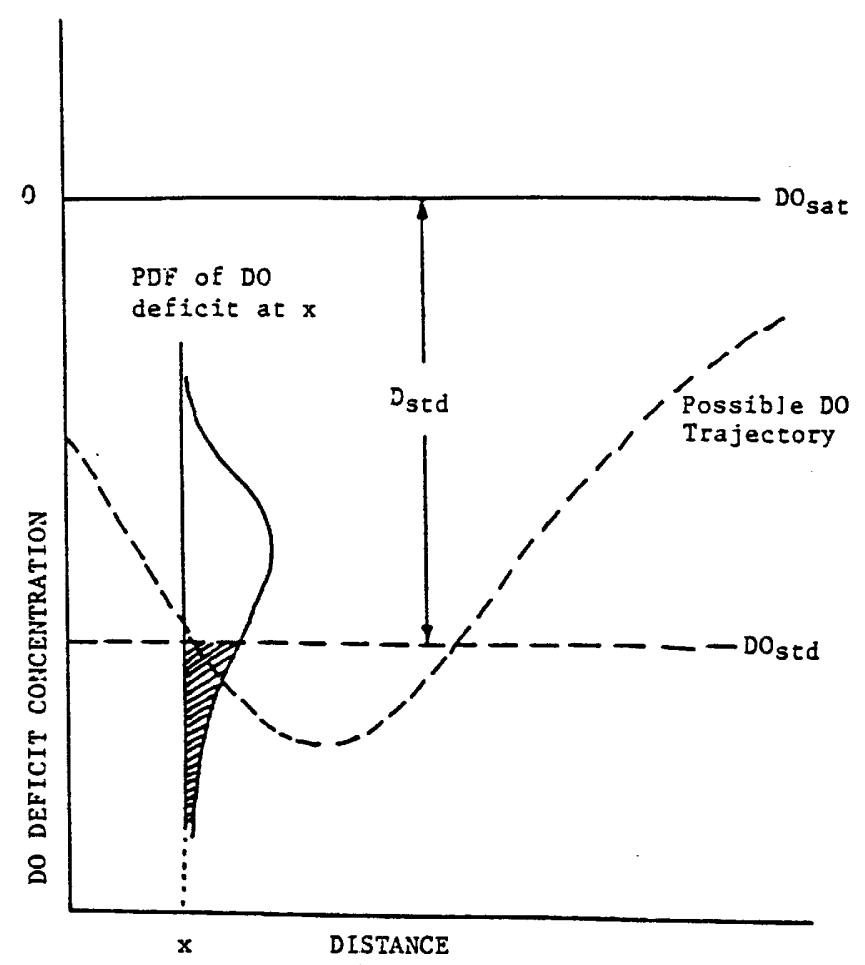

Fig. 1. Schematic diagram of the PDF for the Do concentration.

To assess the values of parameters in a water quality model, data from field measurements and laboratory experiments are analyzed. Parameter uncertainty arises from the estimation of water quality parameters based on a limited amount of data. Furthermore, imprecision and bias in measurements contribute to the total uncertainty associated with the parameters. For instance, parameters such as $K_{\mathrm{a}}, K_{\mathrm{d}}, U, L_{0}$, and $D_{0}$ in (1) are subject to uncertainty.

With the presence of these uncertainties, the actual Do deficit concentration cannot be predicted with certainty. Hence, Do concentration $C_{x}$ or deficit concentration $D_{x}$ could be treated as random variables associated with a probability distribution (see Fig. 1). The cross-hatched area under the probability density function (PDF) of DO concentration represents the risk of violating the specified DO standard.

\section{RISK OF VIOLATING DO STANDARD}

Mathematically, the risk of violating the Do standard, $C_{\text {std }}$, at a given location of stream can be expressed as:

$\alpha=\operatorname{Pr}\left[C_{x}<C_{\text {std }}\right]$ 
or alternatively

$\alpha=\operatorname{Pr}\left[D_{x} \geqslant D_{\text {std }}\right]$

in which $\alpha$ is the risk, $\operatorname{Pr}\left[\right.$ is a probability operator, and $D_{\text {std }}$ is the allowable deficit to standard: $D_{\text {std }}=C_{\text {sat }}-C_{\text {std }}$.

In risk/reliability computations, a performance variable $W$ is commonly used to indicate the state of the system: failure $(W<0)$ or safe $(W \geq 0)$. Based on (4), the performance variable $W$ can be defined as:

$W=D_{\text {std }}-D_{x}\left(K_{\mathrm{a}}, K_{\mathrm{d}}, U, L_{o}, D_{0}\right)$

The performance variable $W$ is also a random variable because it is a function of random water quality parameters. In terms of performance variabe $W$ the risk of violating Do standard can be expressed as:

$\alpha=\operatorname{Pr}[W<0]=F_{Z}(-\beta)=1-F_{Z}(\beta)$

in which $F_{Z}(\quad)$ is the cumulative distribution function (CDF) of standardized performance variable $W$, i.e.

$Z=\left(W-\mu_{W}\right) / \sigma_{W}$

where $\mu_{W}$ and $\sigma_{W}$ are the mean and standard deviation of $W$, respectively. The argument $\beta=\mu_{W} / \sigma_{W}$ in the CDF is the reliability index. The corresponding water quality compliance reliability increases as the value of $\beta$ gets larger.

\section{METHODS FOR EVALUATING RISK OF VIOLATING DO STANDARD}

In this section, methods that are useful for computing the risk of violating Do standard are briefly described and discussed.

Direct integration. Referring to (2), the shape of PDF's of $D_{x}, C_{x}$ and $W$ are identical when $C_{\text {sat }}$ and $C_{\text {std }}$ are constants. The difference lies in the locations of the respective means. Hence, knowing the PDF of one of the three random variables would know the PDF's of the other two. If the PDF of deficit concentration $D_{x}$ or performance variable $W$ is known, the risk of violating Do standard can be obtained by carrying out the following integration:

$\alpha=\int_{D_{\text {std }}}^{C_{\text {sat }}} f_{\mathrm{Dx}}(u) \mathrm{d} u=\int_{-C_{\text {std }}}^{0} \mathrm{f}_{W}(w) \mathrm{d} w$

in which $f_{D x}(\cdot)$ and $f_{W}(\cdot)$ are the PDF's of deficit concentration and performance variable, respectively.

To implement equation (7), difficulty arises regarding the identification of the PDF's of $D_{x}$ or $W$. Due to the highly nonlinear relationship between 
deficit concentration and water quality parameters, analytical derivation of the exact PDF of $W$ or $D_{x}$ is a formidable task in most situations. Recently, Tung and Hathhorn (1988) examined the appropriateness of some commonly used probability distributions in describing the random characteristics of Do deficit concentration computed by (1) in that various distributions for water quality parameters were considered. It was found that in a great majority of the cases investigated, a two-parameter lognormal distribution yielded the best fit, and gamma distribution was the second best.

Simulation approach. Monte Carlo simulation is the general purpose method to estimate the statistical properties of a random variable $Y$ that is related to several random variables $X=\left(X_{1}, X_{2}, \ldots, X_{n}\right)$ in a general function as $Y=g(X)$. Referring to (1), vector $X$ consists of water quality parameters and $Y$ corresponds to $D_{x}$. In Monte Carlo simulation, the values of water quality parameters are generated according to their distributional properties. The generated water quality parameter values are used in (1) to compute the corresponding Do deficit concentration. After a large number of sample realizations of Do deficit concentration are generated, the risk of violating the specified DO standard can be estimated as the percentage of the number of realizations that $D_{x}$ exceeds $D_{\text {std }}$.

The major disadvantage of Monte Carlo simulation is its computation intensiveness. The number of sample realizations required in simulation to accurately estimate the risk depends on the magnitude of the unknown risk itself. In general, as the risk gets smaller the required number of simulated realizations increases. Therefore, some variations of Monte Carlo simulation to accurately estimate the risk, while keeping the computation down, are developed. They include stratified sampling and Latin hypercubic sampling (McKay et al., 1979), importance sampling (Mazumdar, et al., 1975; Harbitz, 1983; Schueller and Stix, 1986), and reduced space approach (Karamchandani, 1987).

Mean-value first-order analysis. From (6), one needs to know $\mu_{W}, \sigma_{W}$, and $F_{Z}$ to compute the exact risk of violating a Do standard. Rather than attempting to derive the true values of $\mu_{W}$ and $\sigma_{W}$, which is generally difficult, the mean-value first-order second-moment (MFOSM) method estimates $\mu_{W}$ and $\sigma_{W}$ by applying Taylor expansion on $W$ with respect to the mean values of water quality parameters. The first-order Taylor series expansion of $W$ can be expressed as:

$$
\begin{aligned}
W= & D_{\text {std }}-W\left(\bar{K}_{\mathrm{a}}, \bar{K}_{\mathrm{d}}, \bar{U}, \bar{L}_{0}, \bar{D}_{0}\right)-S_{K_{\mathrm{a}}}\left(K_{\mathrm{a}}-\bar{K}_{\mathrm{a}}\right) \\
& -S_{K_{\mathrm{d}}}\left(K_{\mathrm{d}}-\bar{K}_{\mathrm{d}}\right)-S_{U}(U-\bar{U}) \\
& -S_{L_{0}}\left(L_{0}-\bar{L}_{0}\right)-S_{D_{0}}\left(D_{0}-\bar{D}_{0}\right)
\end{aligned}
$$


in which the over-bar on each water quality parameter represents the mean value of the corresponding parameter, $S_{x}$ is the sensitivity coefficient of the random water quality parameter $X$ which is the first-order partial derivative of the performance variable with respect to each individual random water quality parameter, $\partial W / \partial X$, evaluated at the mean of water quality parameters. Expressions for sensitivity coefficients in (8) based on (1) can be found elsewhere (Chadderton et al., 1982; Hathhorn and Tung, 1987). The MFOSM method approximates the true mean and variance of performance variable $W$ by applying expectation and variance operators to (8). The results can be written as:

$$
\begin{aligned}
\mu_{\mathrm{W}} \approx D_{\mathrm{std}} & -W\left(\bar{K}_{\mathrm{a}}, \bar{K}_{\mathrm{d}}, \bar{U}, \bar{L}_{0}, \bar{D}_{0}\right) \\
\operatorname{Var}[W] \approx & S_{K_{\mathrm{a}}}^{2} \operatorname{Var}\left[K_{\mathrm{a}}\right]+S_{K_{\mathrm{d}}}^{2} \operatorname{Var}\left[K_{\mathrm{d}}\right]+S_{U}^{2} \operatorname{Var}[U] \\
& +S_{L_{0}}^{2} \operatorname{Var}\left[L_{0}\right]+S_{D_{0}}^{2} \operatorname{Var}\left[D_{0}\right] \\
& +2 S_{K_{\mathrm{a}}} S_{U} \operatorname{Cov}\left[K_{\mathrm{a}}, U\right]
\end{aligned}
$$

in which $\operatorname{Var}[$ ] and Cov[ ] are the variance and covariance operators, respectively. Note that, in (10), only the correlation between $K_{\mathrm{a}}$ and $U$ is considered in this study.

To compute the risk of violating of DO standard, an appropriate probability distribution for the performance variable $W$ is selected. Based on the results of a numerical experiment by Tung and Hathhorm (1988), one can use lognormal or gamma distribution to compute the risk. It should be recognized that the risk so computed is only an approximation to the true value.

The use of MFOSM method in evaluating the risk analysis has several advantages (Yen et al., 1986) including:

(1) the technique is simple in usage and flexible to accommodate practically any problem;

(2) it explicitly accounts for uncertainties in the problem;

(3) its demand on data information is moderate which primarily needs the mean and variance of parameters with uncertainty; and

(4) it provides insight to the relative significance of contributing parameters subject to uncertainty.

On the other hand, the MFOSM method possesses some weaknesses:

(1) inability to handle distributions with large skew coefficient;

(2) generally poor estimation of the mean and variance of nonlinear function; and

(3) sensitivity of the computed risk to the formulation of performance variable. 
To reduce the effect of nonlinearity, one method is to include the second-order terms in Taylor expansion. This would increase the burden of analysis by having to compute the second-order partial derivatives and higher-order statistical moments, which may not be so easily and reliably obtained. The alternative is to use the advanced first-order analysis described in the next section.

Advanced first-order second-moment (AFOSM) method. The main thrust of the AFOSM analysis is to eliminate the disadvantages of the MFOSM method, while keeping the advantages of the first-order approximation. The difference of AFOSM method is that the expansion point on the critical surface is taken in the first-order Taylor series. That is, the critical point $x^{*}=\left(K_{\mathrm{a}}^{*}, K_{\mathrm{d}}^{*}, U^{*}, L_{0}^{*}, D_{0}^{*}\right)$ must satisfy:

$W\left(K_{\mathrm{a}}^{*}, K_{\mathrm{d}}^{*}, U^{*}, L_{0}^{*}, D_{0}^{*}\right)=0$

Taking the critical point $x^{*}$ as the expansion point, the mean and variance (under the condition of independence of random water quality parameters) of the performance variable $W$ can be obtained by replacing the average water quality parameters in (9) and (10) by $x^{*}$. Therefore, the mean, variance, sensitivity coefficients of $W$, and the reliability index $\beta^{*}=\mu_{W}^{*} / \sigma_{W}^{*}$ are functions of the unknown critical point.

Equation (11) can have an infinite number of critical points satisfying it. From the system performance viewpoint, one is more concerned with the combination of random parameters that would yield the highest system risk. Hence, the main task of the AFOSM method is to identify the worst critical point yielding the highest risk of violating a water quality standard. Referring to (6), the risk of violating the DO standard is a monotonic decreasing function of the reliability index $\beta^{*}$. The task to determine the critical point $\left(K_{\mathrm{a}}^{*}, K_{\mathrm{d}}^{*}, U^{*}, L_{0}^{*}, D_{0}^{*}\right)$ that maximizes the risk of violating a Do standard is equivalent to minimizing the value of reliability index $\beta^{*}$. Therefore, the evaluation of risk by the AFOSM method is a problem of solving a constrained nonlinear minimization problem.

An iterative algorithm is described by Ang and Tang (1984) for determining the worst critical point. Numerical experiences indicate that the algorithm does not necessarily converge. It becomes more troublesome when the risk get smaller. An alternative is to cast the problem into an optimization framework. Yen et al. (1986) proposed solving the following model for determining the worst critical point:

Minimize $\left|W\left(x^{*}\right)\right|$

Subject to

$X_{i}^{*}+\delta_{i}^{*} \sigma_{i} \beta^{*}=\mu_{i} \quad$ for $\mathrm{i}=1,2, \ldots, n$

$l_{i} \leq x_{i}^{*} \leq u_{i} \quad$ for $\quad \mathrm{i}=1,2, \ldots, n$ 
in which $x^{*}$, for simplicity, represents the vector of random water quality parameters in (1), $\mu_{i}$ and $\sigma_{i}$ are the mean and standard deviation of the $i$ th water quality parameter, respectively, $l_{i}$ and $u_{i}$ are the lower and upper bounds of random variable $X_{i}$, respectively, and $\delta_{i}^{*}$ is called the directional derivative for $X_{i}=x_{i}^{*}$ which can be computed as:

$\delta_{i}^{*}=\mathrm{S}_{i}^{*} \sigma_{i} / \sigma_{W}^{*}$

In fact, directional derivative $\delta_{i}^{*}$ is also a function of unknown critical failure point. The solution to (12)-(14) can be obtained by applying a constrained nonlinear optimization procedure such as the generalized gradient method (Lasdon et al. 1982). The optimal solution to (12)-(14) yield the worst combination of feasible water quality parameters along with the corresponding minimum reliability index value. The risk of violating a DO standard can be computed by $(6)$ if the probability distribution of the performance variable $\mathrm{W}$ is given or known. Applications (Yen et al., 1986) of the AFOSM method indicate that the use of a normal distribution provide sufficiently accurate estimation of the risk.

The above description of AFOSM method is suitable in cases where all parameter in a water quality model independently normal random variables. However, in reality, parameters in a water quality model may be non-normal and skewed. When non-normal random variables are involved, transformation to the equivalent normal variables can be made. Rackwitz (1976) proposed an approach to transform a non-normal distribution into an equivalent normal distribution. A table of normal transformation of some commonly used non-normal distributions is given by Yen et al. (1986). To incorporate normal transform into the AFOSM method, one simply replaces $\mu_{i}$ and $\sigma_{i}$ in (12)-(14) by $\mu_{i N}$ and $\sigma_{i N}$ which are, respectively, the mean and standard deviation corresponding to the equivalent normal distribution at the failure point $x_{i}^{*}$.

The AFOSM method can also handle correlated random variables. When some of the random variables involved in the performance function are correlated, transformation of correlated variables to uncorrelated ones is first made before the step of normal transform is taken. Detailed discriptions on how to deal with correlated data in the AFOSM are given by Ang and Tang (1984) and Yen and Tung (1989).

\section{NUMERICAI EXAMPLE AND COMPARISON OF METHODS}

The methods described above, except direct integration, are applied to compute the risk of violating DO standards. Direct integration method is not considered because the exact statistical properties of deficit concentration are not known. The emphasis here is to examine the performance of the two 
TABLE 1

Statistical properties of water quality parameters used in the numerical example

\begin{tabular}{lrll}
\hline Model Parameters & Mean & $\begin{array}{l}\text { Standard } \\
\text { deviation }\end{array}$ & Units \\
\hline$K_{\mathrm{d}}$ & 0.35 & 0.10 & day $^{-1}$ \\
$K_{\mathrm{a}}$ & 0.70 & 0.20 & day $^{-1}$ \\
$U$ & 10.00 & 3.00 & miles $/$ day \\
$L_{0}$ & 18.00 & 5.00 & $\mathrm{mg} / \mathrm{L}$ \\
$D_{0}$ & 1.00 & 0.30 & $\mathrm{mg} / \mathrm{L}$ \\
\hline
\end{tabular}

first-order second-moment methods. Since the true probability distribution of deficit concentration cannot be easily derived, the Monte Carlo simulation is used to generate the distribution of the deficit concentration. The simulation generated distribution is used as the 'true' one for comparing the relative accuracy of the two first-order second-moment methods.

The mean and standard deviation of the water quality parameters used are given in Table 1 . The location at which the risk of violating Do standard is evaluated is 10 miles downstream of the initial condition. To investigate the effect of correlation among water quality parameters, two cases are considered with respect to the correlation of water quality parameters. One case considers that all water quality parameters are uncorrelated, while the other uses a correlation coefficient of 0.8 (based on Issacs et al., 1969) between reaeration coefficient $K_{\mathrm{a}}$ and average flow velocity $U$. Distributions of water quality parameters are assumed to be either all normal or all lognormal.

The probabilities of violating various levels of DO standard computed by the two first-order methods, along with the simulation results, are given in Tables 2 and 3. It should be mentioned that the risks of violating Do standards computed by the MFOSM method are not affected by the distribution of water quality parameters for the fixed mean and variance. However, the risk is dependent on the distribution of the performance variable. This is why the risks computed by the MFOSM method are identical in Table 2 (a) and 3 (a); so are the risks in Table 2 (b) and 3 (b). Different correlation assumptions will affect the mean value of Do deficit computed by the MFOSM method. The last columns of both Tables 2 and 3, which were obtained from the Monte Carlo simulations, are used as the basis for comparing the accuracy of the two first-order methods. It is evident that the AFOSM method out-performs the MFOSM method, especially when the value of deficit to Do standard increases. Furthermore, the performance of the AFOSM method is superior to the MFOSM method 
TABLE 2

Risks of violating Do standards when all water quality parameters are normally distributed

(a) All water quality parameters are independent

\begin{tabular}{lllll}
\hline $\begin{array}{l}\text { Deficit to } \\
\text { standard }\end{array}$ & MFOSM & & AFOSM & SIMUL \\
\cline { 2 - 4 } & Normal & Lognormal & & \\
\hline $2.0 \mathrm{mg} / \mathrm{L}$ & 0.967 & 0.996 & 0.968 & 0.948 \\
$3.0 \mathrm{mg} / \mathrm{L}$ & 0.881 & 0.913 & 0.821 & 0.778 \\
$4.0 \mathrm{mg} / \mathrm{L}$ & 0.695 & 0.663 & 0.565 & 0.509 \\
$5.0 \mathrm{mg} / \mathrm{L}$ & 0.440 & 0.367 & 0.317 & 0.268 \\
\hline
\end{tabular}

(b) Correlation coefficient between $K_{\mathrm{a}}$ and $U$ is 0.8

\begin{tabular}{lllll}
\hline $\begin{array}{l}\text { Deficit to } \\
\text { standard }\end{array}$ & MFOSM & AFOSM & SIMUL \\
\cline { 2 - 4 } & Normal & Lognormal & & \\
\hline $2.0 \mathrm{mg} / \mathrm{L}$ & 0.965 & 0.996 & 0.964 & 0.949 \\
$3.0 \mathrm{mg} / \mathrm{L}$ & 0.881 & 0.916 & 0.810 & 0.783 \\
$4.0 \mathrm{mg} / \mathrm{L}$ & 0.712 & 0.684 & 0.561 & 0.530 \\
$5.0 \mathrm{mg} / \mathrm{L}$ & 0.476 & 0.413 & 0.330 & 0.304 \\
\hline
\end{tabular}

when the distribution of water quality parameters is non-normal. The accuracy in estimating risk of violating Do standard by the MFOSM deteriorates rather rapidly as the risk level decreases.

TABLE 3

Risks of violating Do standards when all water quality parameters are lognormally distributed (a) All water quality parameters are independent

\begin{tabular}{lllll}
\hline $\begin{array}{l}\text { Deficit to } \\
\text { standard }\end{array}$ & MFOSM & & AFOSM & SIMUL \\
\cline { 2 - 5 } & Normal & Lognormal & & \\
\hline $2.0 \mathrm{mg} / \mathrm{L}$ & 0.967 & 0.996 & 0.988 & 0.977 \\
$3.0 \mathrm{mg} / \mathrm{L}$ & 0.881 & 0.913 & 0.820 & 0.797 \\
$4.0 \mathrm{mg} / \mathrm{L}$ & 0.695 & 0.663 & 0.514 & 0.497 \\
$5.0 \mathrm{mg} / \mathrm{L}$ & 0.440 & 0.367 & 0.269 & 0.250 \\
\hline
\end{tabular}

(b) Correlation coefficient between $K_{\mathrm{a}}$ and $U$ is 0.8

\begin{tabular}{lllll}
\hline $\begin{array}{l}\text { Deficit to } \\
\text { standard }\end{array}$ & MFOSM & AFOSM & SIMUL \\
\cline { 2 - 5 } & Normal & Lognormal & & \\
\hline $2.0 \mathrm{mg} / \mathrm{L}$ & 0.965 & 0.996 & 0.972 & 0.975 \\
$3.0 \mathrm{mg} / \mathrm{L}$ & 0.881 & 0.916 & 0.801 & 0.799 \\
$4.0 \mathrm{mg} / \mathrm{L}$ & 0.712 & 0.684 & 0.513 & 0.519 \\
$5.0 \mathrm{mg} / \mathrm{L}$ & 0.476 & 0.413 & 0.280 & 0.285 \\
\hline
\end{tabular}


Because of the existence of various uncertainties in stream environments, the impact of water quality decision should be assessed using probabilitistic methodologies. In a stochastic stream environment, the evaluation of the probability of violating the specific water quality standard is one of the important exercise in water quality monitoring and regulation. This paper discusses several methods of computing the risk of violating the DO standard in a stream using the Streeter-Phelps equation. The techniques can be applied to other water quality indicators along with appropriate mathematical models. Comparisons of two first-order methods, namely AFOSM and MFOSM, are made regarding their accuracies in computing the risk of violating Do standard. It is found that when the risk level is high, the two methods yield rather compatible results. However, as the risk level decreases the AFOSM method is superior to the MFOSM method.

In places where water quality management decisions are carefully made, the risk of having negative events happen is usually small. To estimate such risk accurately, it is recommended to use the more sophisticated AFOSM method. The use of MFOSM method, based on the Streeter-Phelps equation, over-estimates the risk of violating the water quality standard. This might lead to an over-conservative management decision which may or may not be desirable.

\section{ACKNOWLEDGEMENT}

The activities on which this report is based were financed in part by the Department of Interior, U.S. Geological Survey through the Wyoming Water Research Center at the University of Wyoming.

\section{REFERENCES}

Ang, A.H.-S. and Tang, W.H., 1984. Probability Concepts in Engineering Planning and Design, Vol. II: Decision, Risk, and Reliability. Wiley, New York, 562 pp.

Chadderton, R.A., Miller, A.C. and McDonnell, A.J., 1982. Uncertainty analysis of dissolved oxygen model. J. Environ. Eng. ASCE, 108: 1003-1023.

Dobbins, W.E., 1964. BOD and oxygen relationships in streams. J. Sanit. Eng. Div. ASCE, 90: 53-78.

Harbitz, A., 1983. Efficient and accurate probability of failure calculation by use of the importance sampling technique. Paper presented 4th Int. Conf. Applications of Statistics and Probability in Soil and Structural Engineering, Univ. Florence, Italy.

Hathhorn, W.E. and Tung, Y.K., 1987. Waste load allocation in stochastic stream environments. Technical report, Wyoming Water Research Center, University of Wyoming, Laramie, WY, 392 pp. 
Hathhorn, W.E. and Tung, Y.K., 1988. Assessing the risk of violating stream water quality standards. J. Environ. Manage., 26: 321-338.

Issacs, W.P., Chulavachana, P. and Bogart, R., 1969. An experimental study of the effects of channel surface roughness on the reaeration rate coefficient. In: Proc. 24th Industrial Waste Conf., 6-8 May 1969, Purdue University, West Lafayette, IN, pp. 1464-1476.

Karamchandani, A., 1987. Structural system reliability analysis methods. Report to Amoco Production Company. Department of Civil Engineering, Stanford University, Standford, CA, 92 pp.

Krenkel, P.A. and Novotny, V., 1980. Water Quality Management. Academic Press, New York, $671 \mathrm{pp}$.

Lasdon, L.S., Warren, A.D. and Ratner, M.W., 1982. GRG2 User's Guide. University of Texas, Austin, TX.

Mazumdar, M., 1975. Importance sampling in reliability estimation. In: Reliability and Fault Tree Analysis, Theoretical and Applied Aspects of System Reliability and Safety Assessment. SIAM, Philadelphia, PA, 927 pp.

McKay, M.D., Beckman, R.J. and Conover, W.J., 1979. A comparison of three methods for selecting values of input variables in the analysis of output from a computer code. Technometrics, 21: 239-245.

Rackwitz, R., 1976. Practical probabilistic approach to design. Bull. 112, Comité European du Beton, Paris.

Schueller, G.I. and Stix, R, 1986. A critical appraisal of methods to determine failure probabilities. Report No. 4-86, Institute fur Mechanik, Univ. Innsbruck, Austria.

Streeter, H.W. and Phelps, E.B., 1925. A study of the pollution and natural purification of the Ohio River. Publ. Health Bull. 146, U.S. Public Health Service, Washington, DC, pp. 127-146.

Tung, Y.K. and Hathhorn, W.E., 1988. Assessment of probability distribution of dissolved oxygen deficit. J. Environ. Eng., ASCE, 114: 14211435.

Yen, B.C. and Tung, Y.K., 1989. Some recent progress in risk and uncertainty analysis for hydraulic structure designs. Proc. Sem. Assessment of Risks and Uncertainties in the Design of Hydraulic Structures, XXIII Congress of IAHR, Ottawa, Ont., Canada, 21-25 August 1989.

Yen, B.C., Cheng, S.T. and Melching, C.S., 1986. First-order reliability analysis. In: B.C. Yen (Editor), Stochastic and Risk Analysis in Hydraulic Engineering. Water Resources Publications, Littleton, CO, pp. 1-36. 